Anales de Geografía de la Universidad Complutense ISSN: 0211-9803

http://dx.doi.org/10.5209/AGUC.72981

\title{
"La Paz que queremos": Geografía legal, imaginación urbana y las formas de la ciudad en La Paz (Bolivia)
}

\author{
Pedro Limón López1; María Dolores Lois Barrio² \\ Recibido: 19 de julio del 2019 / Enviado a evaluar: 1 de noviembre del 2019 / Aceptado: 15 de diciembre del 2020
}

\begin{abstract}
Resumen. El objetivo de este artículo es acercarnos al proceso de producción de los imaginarios sobre el espacio público en el planeamiento urbanístico de la ciudad de La Paz (Bolivia). Para ello, recurriremos a la geografía legal, como marco teórico que analiza las interacciones entre las normativas de las instituciones públicas y la planificación del espacio; nuestra hipótesis es que los imaginarios geográficos que proyectan dichos documentos construyen representaciones espaciales de la ciudad y definen los usos del territorio urbano. En términos de metodología, realizaremos un análisis de contenidos de "La Paz que queremos", plan integral urbano que abarcaría de 2015 a 2040. Para dicho análisis, trabajaremos sobre tres líneas de significado: por un lado, las diferentes escalas presentes en la planificación urbana de la ciudad; por otro, las imaginaciones cartográficas urbanas proyectadas desde el documento; y finalmente, en torno a las narrativas sobre Bolivia y lo urbano, para cerrar con una breve discusión en torno a las representaciones de lo urbano, los poderes públicos y la imaginación geográfica del espacio de las ciudades.
\end{abstract}

Palabras clave: Geografía legal; imaginarios urbanos; La Paz (Bolivia).

\section{[en] "La Paz We Want": Legal Geography, Urban Imagination and the Forms of the City in La Paz (Bolivia)}

Abstract. The aim of this article is to reflect on the making of imaginaries about the public space in the urban planning of the city of La Paz (Bolivia). To do this, we will turn to legal geography, as a theoretical framework that analyses the interactions between the regulations of public institutions and space planning. Our hypothesis is that the geographical imaginaries that project these documents construct spatial representations of the city and define the uses of the urban territory. In terms of methodology, we propose an analysis of the contents of "La Paz que queremos", an integral urban plan that would cover 2015 to 2040. For this analysis, we will work on three lines of meaning: on the one hand, the different scales present in the planning urban city; on the other, the urban cartographic imaginations projected from the document; and finally, around the narratives about Bolivia and the urban,

Universidad Internacional de La Rioja (UNIR). España.

2 Facultad de Ciencias Políticas y Sociología. Universidad Complutense de Madrid. España.

E-mail: mdlois@ucm.es 
to close with a brief discussion about the representations of the urban, the public policies and the geographical imagination of the space for the cities.

Keywords: Legal geography; urban geographical imagination; La Paz (Bolivia).

[fr] "La Paz que nous voulons": géographie juridique, imagination urbaine et formes de la ville à La Paz (Bolivie)

Résumé. L'objectif de cet article est d'aborder le processus de production des imaginaires sur l'espace public dans l'urbanisme de la ville de La Paz (Bolivie). Pour ce faire, nous nous tournerons vers la géographie juridique, en tant que cadre théorique analysant les interactions entre la réglementation des institutions publiques et la planification de l'espace; Notre hypothèse est que les imaginaires géographiques qui projettent ces documents construisent des représentations spatiales de la ville et définissent les utilisations du territoire urbain. En termes de méthodologie, nous effectuerons une analyse du contenu de "La Paz que queremos", un plan d'urbanisme intégral qui couvrirait les années 2015 à 2040. Pour cette analyse, nous travaillerons sur trois axes de sens: d'une part, les différentes échelles présentes dans le planning ville urbaine; de l'autre, les imaginations cartographiques urbaines projetées à partir du document; et enfin, autour des récits sur la Bolivie et l'urbain, pour conclure par une brève discussion sur les représentations de l'urbain, les pouvoirs publics et l'imaginaire géographique de l'espace des villes.

Mots Clès: Géographie juridique; l'imaginaire géographique de l'urbain; La Paz (Bolivie).

Cómo citar. Limón López, P; Lois Barrio, M.D. (2020): "La Paz que queremos": Geografía legal, imaginación urbana y las formas de la ciudad en La Paz (Bolivia). Anales de Geografía de la Universidad Complutense, 40(2), 419-437.

Sumario. 1. Introducción. 2. El Marco Teórico-Metodológico: La Geografía Legal Crítica. 3. El caso: del Plan Integral de la Paz 2040 ("La Paz que queremos") al Plan de Centralidades Urbanas. 3.1 Las bases de la planificación urbana de La Paz. 3.2 Imaginación cartográfica y representaciones del espacio urbano en La Paz y en Bolivia. 4. Conclusiones: reconfigurando la ciudad e imaginando lo urbano. 5. Referencias bibliográficas.

\section{Introducción}

Los debates académicos en torno al objeto de estudio de la teoría urbana en general y al concepto de ciudad en particular son una constante en las ciencias sociales, puntualmente actualizados por la aparición de nuevas perspectivas de investigación. En ese sentido, son muchos los trabajos que llaman la atención sobre estas cuestiones $\mathrm{y}$, en particular, sobre los límites de la teoría urbana para conceptualizar las formas cambiantes de las ciudades contemporáneas. Es el caso, por ejemplo, de un artículo clave para este debate que señalaba las limitaciones tanto de la perspectiva postcolonial como de la teoría del Actor-Red y los ensamblajes urbanos para demarcar las lógicas de los procesos de urbanización respecto a las lógicas de otros procesos sociales (Scott y Storper, 2014). El texto propone, precisamente, identificar lo que es propio de lo urbano desde un punto de vista estructural, para poder desarrollar marcos explicativos desde los que entender los elementos comunes del objeto de estudio en los estudios urbanos, entendiendo que "las ciudades, desde la 
Antigüedad hasta hoy, han funcionado como sistemas de densas interacciones locales imbricadas en movimientos complejos y a larga distancia de personas, bienes e información" (Scott y Storper, 2014, p.7). Este texto dio lugar a varios artículos de respuesta y comentarios, entre otras cosas, por poner de manifiesto que no todo lo que ocurre en las ciudades puede ser descrito como intrínsecamente urbano (13: cursiva en original). Y esa sería precisamente una de las propuestas de partida de este artículo: intentar reflexionar sobre las prácticas que se engloban bajo la forma ciudad, entendiendo que pueden registrarse formas constantemente cambiantes, probablemente difusas y frecuentemente paradójicas en torno a lo urbano.

En otra aportación al mismo debate, Wachsmuth (2014) proponía desarrollar una perspectiva crítica a las categorías de urbanización desde las que se mide y observa el proceso a nivel global. En su planteamiento, el concepto de ciudad es teorizado como una "representación ideológica del proceso de urbanización" (Wachsmuth, 2014, p.77: en cursiva en el original), que oscurecería la multiplicidad y variedad de formas de lo urbano. Así, el autor enuncia a tres tropos que serían esenciales para comprender lo que se entiende tanto por procesos de urbanización y la ciudad de forma generalizada (Waschumth, 2014, p. 80), a saber: la oposición campo - ciudad; la concepción de la ciudad como un sistema autoreferente y la consideración de "la ciudad" como un tipo ideal. La discusión teórico-empírica de estas tres dimensiones le permite al autor poner de manifiesto cómo la ciudad se habría convertido en una representación homogénea y mítica de un momento en los procesos de urbanización (Wachsmuth, 2014). Y en esa línea de reflexión situamos la segunda propuesta que querríamos extraer de un acercamiento a este debate: existiría una cierta tendencia a comprender la ciudad como una forma cultural, con un horizonte normativo marcado por un tipo ideal de urbanización. En contraposición a esta forma de acercamiento a las cuestiones urbanas, y siguiendo a Wachsmuth, nuestra idea es poner en valor el acercamiento a la ciudad como práctica, y a la práctica de lo urbano, es decir, a los lugares donde se generan, resisten, construyen o cuestionan lo urbano y la ciudad, con el objetivo de encontrar así interrogaciones en torno a las políticas urbanas y su práctica en torno a las heterogéneas formas de vivir la ciudad. Para hacerlo, recurriremos a la imaginación geográfica, entendida como "la sensibilidad hacia la importancia del lugar, el espacio y el paisaje en la formación y conducta de la vida social (...), que permite a los individuos reconocer la función del espacio y del lugar en su propia vida, en relación con los espacios que ve a su alrededor" (Johnston et al. 2000, p. 321). En otras palabras, nos acercaremos a la interpretación y significación de narrativas e imaginarios del entorno y al modo en que distintos agentes (en este caso, políticos) formulan esas representaciones en sus modos de imaginar y actuar lo urbano. Nuestro objetivo no es reinventar conceptos o añadir apellidos a las ya múltiples categorizaciones sobre la ciudad, ni encontrar el patrón analítico definitivo que englobe la heterogeneidad de las formas urbanas actuales, o llegar a disertar sobre cada ciudad como micro-cosmos específico; lo que pretendemos es ahondar, en la medida de lo posible y desde estudios de caso, en la reflexión en torno a las formas valorativas sobre espacio y modelos de ciudad asociadas con la planificación urbana, 
que en alguna manera parecerían excluir de las investigaciones sobre lo urbano a ciertas prácticas que ocurren en la ciudad.

Así, el objetivo de este artículo es analizar la imaginación geográfica urbana institucionalmente proyectada sobre la ciudad de La Paz (Bolivia). Partiendo de la geografía legal como herramienta analítica, nos acercaremos al plan integral urbano "Plan Integral La Paz 2040: La Paz Que Queremos" concebido como instrumento de reestructuración urbana a desarrollarse entre 2015 y 2040 por parte de la municipalidad boliviana. A partir de un análisis de contenidos, primero se sintetizarán las bases de la planificación, para luego continuar un acercamiento a las representaciones del espacio urbano de La paz. El texto concluye con una serie de reflexiones preliminares en torno a los significados de lo urbano y de la ciudad en La Paz formulados en su documento rector como posibles bases de investigaciones futuras.

\section{EI Marco Teórico-Metodológico: La Geografía Legal Crítica}

El acceso a los espacios públicos ha sido siempre un tema de debate y conflicto en el análisis y la ejecución política en ámbitos urbanos. Además de sus posibilidades de uso y de representación, con frecuencia se establecen mecanismos de distinción, inclusión o exclusión social en función de quién, y dónde, tiene acceso de forma particular a determinados lugares del espacio público (Young y Keil, 2010). En este sentido, cualquier forma de reestructuración y planificación urbana, sea de zonas concretas, sea de un territorio considerado como un todo en conjunto, suponen una reconfiguración de los espacios públicos de la ciudad, del acceso y uso al mismo, así como de las formas en que se representa e imagina dicho espacio. Por dicho motivo, considerando que también existen otras formas de organización y uso de los ámbitos urbanos sujetos a reestructuración, las políticas públicas se erigen como núcleo central de la actuación institucional y del foco de análisis, fundamentalmente en lo relativo a la producción normativa y jurídica.

Durante las últimas tres décadas, en paralelo a las transformaciones urbanas globales, se ha producido una ampliación de las intervenciones políticas mediante los instrumentos jurídicos, al igual que una sujeción a Derecho de múltiples prácticas sociales que anteriormente no estaban sometidas a regulación alguna. Por otro lado, se han consolidado distintos instrumentos ligados a la securitización y políticas de seguridad que han supuesto un paso "de las políticas diseñadas a promover la integración social (...) a otras basadas en la asimilación cultural, la neutralización política o la exclusión social, que se han incrementado al basarse en la creencia de amenazas y riesgos exteriores, así como la prevención de los mismos" (Banakar, 2010, pp.12-13). En torno a estas transformaciones los espacios urbanos han sido fundamentales, especialmente mediante las políticas desarrolladas alrededor de los programas de regeneración urbana, a través de los cuales se ha producido un cambio de las representaciones e imaginarios políticos, así como sobre los espacios públicos, 
incrementando la atención académica sobre el efecto que ejercen las distintas normas en los ámbitos públicos (Lippens, 2004).

La primera disciplina que centró su atención en la producción normativa como objeto de estudio fue la Sociología jurídica, que es "un campo multidisplinar formado por diferentes aproximaciones al estudio del derecho en sociedad (...), y sostiene que todo el derecho y sus manifestaciones deberían estudiarse empírica y contextualmente" (Banakar, 201, p. 2). El Derecho es utilizado como un punto de partida desde el cual se analizan una serie de cuestiones teóricas y empíricas que enmarcan, ocultan o incluso proyectan algunos de los conflictos e interrogantes políticos principales, aunque esta perspectiva no prestaba atención al espacio como dimensión social, obviando diatribas esenciales relativas al espacio público o al análisis entrelazado de la legislación, el espacio y la imaginación geográfica. Siguiendo a Blomley (2008, p. 156), hay múltiples nexos espacio-legales que no pueden omitirse: los dos elementos se producen de forma recíproca -son causa y efecto de las mismas relaciones sociales-; la espacialidad siempre da forma y es reformulada por la praxis legal; el espacio siempre es un imaginario para el Derecho y la aplicación efectiva de los instrumentos jurídicos, creando "las formas en que éstos se constituyen, contestan y funcionan para producir, y operar desde, espacios delimitados» (Blomley, 2008: 158), mientras que el tipo de categorización legal es un modo concreto de espacialización (Braverman et al., 2013; Bennett y Layard, 2015; Delaney, 2015a, 2015b).

La pregunta principal, entonces, se ciñe en torno a qué concepción del espacio de la ciudad produce, y es reproducida por la actuación legal, en este caso, el Plan Integral de La Paz, así como qué imaginarios del espacio urbano son proyectados e imaginados, para lo cual es pertinente la aplicación de la Geografía Legal. Constituida por distintas corrientes teóricas, la Geografía crítica del Derecho se distingue por rechazar que el espacio y la normativa sean considerados entes aislados entre sí, sumando problemáticas de investigación conforme a su evolución histórica como perspectiva analítica. En un principio, vinculado al enfoque de la Geografía Regional del Derecho, los estudios se restringían al análisis estatal que, desde una perspectiva de determinismo ambiental, tenían autores como Bodin o Montesquieu o, ya en el siglo XX, al enfoque de Wigmore, que fue pionero en analizar la influencia de la Geografía sobre el Derecho, realizándose tipologías de Estados que se incluían dentro de clasificaciones conforme a variables ligadas a la Geografía Física. A mediados de los años treinta del siglo XX -a instancias de Whittlesey esencialmente-, se invirtió la relación, analizando qué efectos produce la autoridad institucional sobre el paisaje, sobre todo mediante el estudio de leyes relativas a la regulación de propiedad privada, recursos o aranceles, investigando cómo influyen los instrumentos normativos sobre el espacio y asumiendo un determinismo normativo que implicó la separación del espacio, la sociedad y el Derecho como objetos de análisis. Finalmente, y frente al determinismo ambiental y normativo respectivamente, apareció una corriente crítica de la Geografía Legal que, a partir de los años noventa, enfatizó en rechazar el espacio y el Derecho como entes separados y pre-políticos (Johnston et al, 2000, pp. 127-130). Desde dicha perspectiva, estos elementos no sólo se complementan, sino 
que están solapados, "examinando cómo el espacio modifica o reforma los efectos de la ley y, simultáneamente, cómo la ley da forma a las relaciones espaciales» (Martin et al., 2010, p. 177).

Desde esta visión, ambas esferas son constitutivas entre sí, centrándose en tres cuestiones: el análisis de los modos a través de los cuales la acción e interpretación legal produce determinados espacios; la naturaleza situada de la interpretación legal; y en el estudio de las demandas geográficas y representaciones contenidas en el discurso legal (Gregory et al, 2009). De esta forma, la Geografía política del Derecho se dirige hacia "diferentes espacios y localizaciones; reglas y expectativas que regulan dichos espacios; y las implicaciones de estas distintas espacialidades sobre las cualidades de los actores públicos" (Staeheli, 2010, p.71).

Finalmente, gran parte de las transformaciones políticas y económicas desarrolladas en el ámbito metropolitano a escala global han sido diseñadas, planificadas e implementadas desde la propia promulgación, aplicación e implementación legal, especialmente al relacionar dichas reestructuraciones urbanas con los procesos de globalización (Blomley, 2003b; Delaney et al., 2010; Barkan, 2011) o con los conflictos existentes por los el uso, acceso y exclusión de diferentes colectivos sociales (Mitchell, 1997, 2003; Forest, 2004; Blomley, 2003a, 2004, 2010; Dangschat, 2009; Mitchell y Heynen, 2009; Herbert, 2010; Staeheli, 2010; Baptista, 2013).

De esta forma, frente a la escasez existente tanto en el contexto latinoamericano como en el sur europeo de estudios comprehensivos y explicativos de las relaciones entre la imaginación geográfica y la elaboración e implementación de la normativa en grandes proyectos de reestructuración urbana, recurriremos a esta herramienta analizar cómo se articulan los usos y representaciones de lo urbano mediante la ley. Por otro lado, el análisis de dicha normativa es básico en la medida en que, a partir de la ley, se produce un tipo específico de comprensión de las formas de espacialización, estableciendo narrativas concretas sobre límites territoriales, interacción social o relaciones de propiedad (Martin et al, 2010). En último término, el estudio de la ley desde una perspectiva geográfica tiene una importancia política central, toda vez que desde aquella se establecen controles institucionales, pero también referentes simbólicos -tanto culturales como políticas- a los que se apela para reformular y utilizar los espacios públicos de la ciudad (Blomley, 2008, pp. 156-157). Dicho de otra forma:

“[...] en la medida en que lo público se constituye parcialmente en espacios públicos, dónde se localizan tales espacios puede ser algo decisivo [...]. Al cerrar determinados espacios a la expresión política [ampliando y potenciando otros], la exclusión de las voces disidentes es ocultada por la apariencia de que el espacio público es políticamente inclusivo [...]. Es así como las políticas del espacio público desplazan quiénes son silenciados al dónde se debe practicar la política [...], [y es así como] las políticas del espacio público pueden dar forma a la naturaleza de lo político en el espacio público" (Mitchell y Staeheli, 2005, p. 798). 
En términos de metodología, este trabajo recurrirá al método del estudio de caso extendido o ampliado, a partir de un análisis de contenidos del documento "La Paz que queremos", plan integral urbano cuyo marco espacial de referencia es la ciudad de La Paz, y cuyo marco temporal abarcaría de 2015 a 2040. Al igual que sucede en la gran mayoría de estudios de este tipo, aunque la particularidad del caso no restringe su capacidad de sistematización, el estudio amplio del contexto resultaría fundamental para este tipo de análisis, como sucede en este caso con el proceso de transformación en Bolivia en las dos últimas décadas. Según el Instituto Nacional de Estadística del país, en una población de 11.216 .000 habitantes, el $69,4 \%$ de la población se concentra en áreas urbanas y el 30,6\% en áreas rurales (Instituto Nacional de Estadística, 2018). Por tanto, la importancia de la práctica de la ciudad en Bolivia sería clave para comprender la forma en la que las instituciones proyectan la urbanidad y las formas de la ciudad en un entorno mayoritariamente urbano y en constante transformación. Así, siguiendo una metodología del análisis crítico del discurso, el estudio se centraría en la producción discursiva práctica (Chouliaraki y Fairclough, 1999, p. 23) del espacio de la ciudad, si bien en este caso nos centramos en el análisis de contenidos desde la planificación urbanística.

\section{El caso: del Plan Integral de la Paz 2040 ("La Paz que queremos") al Plan de Centralidades Urbanas}

La Ley Marco de Autonomías y Descentralización “Andrés Ibáñez” (ley número 031), promulgada el 19 de julio de 2010 es una de las cinco leyes que regula la organización y planificación territorial del Estado Plurinacional de Bolivia. En su artículo 131, establece que:

"I. La planificación integral consolida la planificación del desarrollo con la organización territorial, articulando en el corto, mediano y largo plazo la economía plural, el uso y la ocupación del territorio y las estructuras organizativas del Estado, e incluye la programación de la inversión, el financiamiento y el presupuesto plurianual II. En este marco, la planificación territorial del desarrollo es la planificación integral para el vivir bien bajo la responsabilidad y conducción de los gobiernos autónomos departamentales, regionales, municipales e indígena originario campesinos, en coordinación con el nivel central del Estado y en articulación con la planificación sectorial."

En este marco, la ciudad de La Paz desarrolla el primer plan municipal largo plazo, partiendo de la Ley Municipal autonómica número 068 del Gobierno autónomo municipal de La Paz, promulgada de forma efectiva a 4 de abril de 2014, donde se aprueba la formulación del Plan de Desarrollo Municipal Integral "Plan La Paz 2040". Este Plan se contempla como integración del Plan de Desarrollo Municipal y el Plan Municipal de Ordenamiento Territorial y Usos del suelo, "guía de las 
transformaciones estructurales y las estrategias de desarrollo del Municipio de La Paz durante los próximos años" (exposición de motivos de la ley). Como forma de salvaguarda institucional, dicho plan forma "parte integrante e indisoluble de la presente Ley Autónoma Municipal" (artículo 3), instando al desarrollo municipal y de ordenación territorial del mismo y estableciendo los ejes estratégicos alrededor de los cuales ha de llevarse a cabo, a saber:

-Sustentabilidad y eficiencia

-Protección, seguridad y resiliencia

-Vida, dinamismo y orden

-Felicidad, interculturalidad e inclusión

-Emprendimiento, innovación y prosperidad

-Autonomía, participación y corresponsabilidad

Dichos ejes producen diversas formas de planificación e imaginación geográfica que, como veremos, no sólo atañen al diseño y estructuración de la trama urbana de la ciudad, sino a la proyección sobre lo urbano y a $\mathrm{La}$ Paz en perspectiva regional, estatal y global.

\subsection{Las bases de la planificación urbana de La Paz}

A partir de un modelo de desarrollo basado en un municipio compacto, policéntrico, integrado, en equilibrio, corresponsable, con desarrollo humano pleno, seguro, intercultural, sustentable, ecoproductivo y con servicios de alta calidad, con gestión del conocimiento y tecnología e impulsor de la Región Metropolitana (Gobierno Autónomo Municipal de La Paz, 2015, pp. 16-17), el "Plan La Paz 2040" establece las bases de la planificación de la ciudad, y que orientarán la vertebración urbana posterior. Uno de sus pilares básicos es ligar el desarrollo de la ciudad al impulso de su región metropolitana, considerada desde el municipio de La Paz hasta su expansión de los entornos del Altiplano, Valles y Amazonas. En palabras del propio plan:

"El desarrollo local de La Paz se basa en el impulso de su región metropolitana para consolidar su posicionamiento regional, nacional e internacional, [que] por su ubicación geográfica estratégica se constituye en el centro económico de la región" (Gobierno autónomo municipal de La Paz, 2015, p. 74).

Esta directriz rectora tiene un efecto esencial en términos de cómo la inserción regional y global de la ciudad influye en la propia planificación urbanística, así como en las adaptaciones de ésta a la propia situación regional y global paceña. A grandes rasgos, podemos diferenciar tres líneas de actuación singulares en este sentido: en primer lugar, una consideración general del diseño urbano y rural a partir de las funciones productivas y de los recursos existentes en las diferentes áreas incluidas en la planificación. En segundo lugar, una línea temática ligada directamente a los ejes principales del propio plan, que desarrollan el planeamiento conforme a dichas 
variables principales. En tercer y último lugar, una espacialización sectorial de las divisiones administrativas y funcionales a partir del rediseño territorial de La Paz y el establecimiento de nodos de interacción e intercambio y de un programa de centralidades urbanas.

a) Respecto a la primera línea de actuación, se centra en el Plan de Uso de Suelos y el Plan de Ocupación del Territorio, a partir de un diseño que reconoce la existencia de ámbitos rurales y urbanos y que clasifica dichas áreas conforme a su función productiva (Gobierno autónomo municipal de La Paz, 2015, pp. 24-31). El plan no sólo reconoce las actividades existentes, sino que proyecta una transformación de parte del municipio y también de estas actividades. Si bien más del $91 \%$ del territorio del municipio de La Paz se considera rural, y dentro del mismo hay una influencia preeminente de la agricultura anual extensiva -más del $51 \%$ de la superficie total del área rural se destina a dicha actividad (Gobierno autónomo municipal de La Paz, 2015 , p. 25)-, se prevé la existencia de un denominado "suelo de estudio" cuya superficie abarca más de 20.000 hectáreas, se encuentra en el radio suburbano y se ha considerado de tratamiento especial, por sus características productivas (Gobierno autónomo municipal de $\mathrm{La} \mathrm{Paz}, 2015$, p. 29). Aunque no está clara aún su configuración final, podría tratarse de un suelo potencialmente urbanizable, que entroncaría con la pretensión de desarrollar los recursos productivos, la infraestructura y los servicios del área rural en gran parte de su extensión, exceptuando las tierras protegidas ${ }^{3}$.

Desde lo que el plan denomina Ocupación del Territorio, se propone una reestructuración político administrativa con la finalidad de "mejorar la administración, planificación y gestión a partir de distritos homogéneos que permitan una mejor gestión pública, participación ciudadana y administración desconcentrada" (Gobierno autónomo municipal de La Paz, 2015, p.32). A partir del establecimiento de once macrodistritos urbanos y tres macrodistritos rurales, se diseña una re-territorialización, en base a criterios de eficiencia, inversión e institucionalización de los elementos de la ocupación territorial. Los factores que orientarían entonces la ocupación del territorio en La Paz serían las unidades territoriales -en términos generales, conforme a su función productiva-; la promoción de un sistema jerárquico de centralidades; un sistema vial estructurado; áreas y sitios de protección natural y paisajística; una estructura de equipamientos y espacio público urbano; y la vertebración de hábitat y vivienda de calidad (Gobierno autónomo municipal de La Paz, 2015, p. 33).

b) La segunda línea de actuación se centra en torno a los seis ejes estratégicos del Plan de La Paz 2040 anteriormente mencionados, y en cómo éstos permiten articular diferentes elementos en la planificación urbanística y la ordenación territorial, especialmente los tres primeros, que suponen más del $90 \%$ del presupuesto municipal

${ }^{3}$ Las "tierras de protección con uso restringido" abarcan el $46.70 \%$ del área rural, e incluye el Espacio natural de conservación del recurso hídrico que, por su importancia no sólo para el suministro de agua sino también para otros recursos -turismo, minería, etc.-, constituye una zona restringida del municipio (Gobierno autónomo municipal de La Paz, 2015, p. 31). 
proyectado (Gobierno autónomo municipal de La Paz, 2015, p. 88). En torno al primer eje - La Paz sustentable y ecoeficiente- se vertebra la gestión integral del Recurso Hídrico, a partir de represas y plantas de tratamientos de aguas, así como una planificación de la sostenibilidad mediante formas de arquitectura verde, complejos metropolitanos de reciclaje, huertos solares o el establecimiento de áreas protegidas, garantizando la conservación del patrimonio natural, de espacios de conservación o de áreas forestales (Gobierno autónomo municipal de La Paz, 2015, pp. 40-45). El segundo eje se centra en la seguridad físico territorial, la seguridad socieconómica y la seguridad individual, y se desarrolla a partir de un acondicionamiento del territorio conforme a la función urbana o rural de vivienda, recreación o trabajo. Plantea como política de desarrollo la creación de bases de atención y prevención de emergencias, así como la consolidación de planes de recuperación de la infraestructura y de servicios afectados por desastres o de medidas de prevención para la disminución del riesgo - la canalización del Río La Paz o el embovedado del Río Orkojahuira, como ejemplos más significativos-. Asimismo, garantiza la provisión de alimentos en el municipio, a partir de una red de almacenamiento y distribución -centros de acopio, centro de faeno metropolitano o red de galerías comerciales- y de microhuertos familiares que en cierta medida incidiría en la consolidación del municipio como una entidad autosuficiente, con dinámicas de abastecimiento relacionadas con la concepción de la ciudad como lugar de recepción y racionalización de la producción agrícola ligada a los usos productivos de la tierra identificados con áreas rurales.

Respecto a la seguridad ciudadana, además de la propuesta de construir una 'cultura de paz', establece un plan de prevención securitaria a través de la iluminación completa de la ciudad y la planificación de un sistema integral para la seguridad ciudadana -fundamentalmente con centros de comando y control o el refuerzo de una red de estaciones policiales- (Gobierno autónomo municipal de La Paz, 2015: 46-53). En torno al tercer eje -La paz viva, dinámica y ordenada- se articula el ordenamiento territorial del plan, en términos integrales y a partir de una dinámica territorial que trasciende el municipio paceño. Como principales elementos, se establecen planes de rehabilitación y renovación urbana, de recuperación del patrimonio y el establecimiento de un espacio público de calidad nucleado por las denominadas centralidades urbanas macrodistritales, que veremos más adelante. Además, enfatiza a necesidad de un plan de movilidad centrado en una infraestructura de transporte ordenado y limpio -haciendo hincapié en el aspecto peatonal, los parques o los sistemas de transporte colectivo, como el proyecto de tren elevado ecológico-, y el desarrollo de un plan de vivienda de interés social digna y accesible o los 'barrios de verdad' y modelos alternativos de asentamiento rural (Gobierno autónomo municipal de La Paz, 2015, pp. 54-61).

El cuarto eje -La paz feliz, intercultural e incluyente- se articula a partir de diferentes hitos y centros urbanos - Casa municipal de la mujer, red de albergues sociales, centros de protección de niños y niñas trabajadoras, escuelas modelo, centros de saberes, centro de atención animal-, así como de servicios vinculados a la 'calidad de vida' - una estructura de hospitales de diferente nivel, lugares de ocio y deporte, redes de escuelas municipales de artes y de bibliotecas municipales- y de 
gestión de la cultura vinculada a la identidad paceña -sea a través del consumo cultural, la recuperación patrimonial o la resignificación de la memoria mediante casas de cultura o museos- (Gobierno autónomo municipal de La Paz, 2015, pp. 6273). En torno al quinto eje emergería no sólo el impulso de la región metropolitana a nivel multiescalar, sino la configuración de centros que impulsen el emprendedurismo y la innovación en clave productiva - casa del emprendedor, conglomerados manufactureros e industriales o el centro municipal de especialización técnica-, así como la diversificación y ampliación de servicios y productos turísticos, articulándose plataformas de promoción al turismo local, un centro internacional de convenciones para el turismo o circuitos turísticos que permitan aprovechar el patrimonio natural (Gobierno autónomo municipal de La Paz, 2015, pp. 74-79). El sexto y último eje La Paz autónoma, participativa y corresponsable- se centra en la institucionalización de diferentes formas de diversidad territorial -identidades, culturas y tribus urbanas, formas de asentamiento comunitario y observatorios municipales populares-, poniendo en valor la institucionalidad y el valor público de los recursos colectivos; asimismo, planea el establecimiento de una cultura participativa a partir de la formación en cultura urbana, una red de educadores urbanos o formas de participación tecnológica (Gobierno autónomo municipal de La Paz, 2015, pp. 80-85).

c) La tercera línea de actuación de la planificación urbanística pasa por una espacialización sectorial de las divisiones administrativas y funcionales a partir del rediseño territorial de $\mathrm{La} \mathrm{Paz}$, desde el establecimiento de nodos de interacción e intercambio y del programa de centralidades urbanas.

En primer lugar, la espacialización sectorial del rediseño urbano se articula a partir de proyectos estratégicos parciales, que abarcarían desde funciones productivas hasta el abastecimiento de servicios públicos. Según el Plan, esta redistritación vertebraría toda una red de nodos de conexión, centralidades socio-espaciales de trabajo, producción, consumo y servicios, que implicaría una especialización sectorial de lugares (centros), estableciendo como prioridad la conexión e interacción de estos lugares entre sí a lo largo del territorio de la región metropolitana. Asimismo, la creación de estos espacios no sólo estaría vinculada de la propia praxis urbana, sino que en algunos casos constituyen lugares de producción de imaginarios y representaciones sobre lo que se considera 'debe ser' la vida cotidiana paceña -por ejemplo, mediante la red de educación urbana o los talleres distritales sobre concienciación sobre diversidad e interculturalidad (Gobierno autónomo municipal de La Paz, 2015, p. 65).

Además, este rediseño territorial implicaría una condensación de los diferentes ámbitos y espacios urbanos y rurales de La Paz bajo la misma estructura institucional municipal; esto es así tanto en los espacios administrativos que se reconocenmacrodistritos urbanos, macrodistritos rurales, unidades territoriales o centralidades urbanas-, como en la inclusión de gran parte de las actividades y prácticas socioculturales en la regulación del gobierno municipal.

Finalmente, y como elemento central de esta línea de actuación de la planificación urbanística en el Plan Integral, podemos destacar el Programa de centralidades urbanas. En el texto se presenta como un sistema jerárquico de centralidades del 
Municipio de La Paz, siendo que "las centralidades urbanas desconectan las actividades del centro histórico de la ciudad para crear y consolidar otros núcleos de desarrollo urbano con roles y funciones definidos de acuerdo con sus vocaciones y potencialidades, estructurando y equilibrando el desarrollo del territorio" (Gobierno autónomo municipal de La Paz, 2015, p. 34). Dicho programa proyecta 19 centralidades, en torno a las cuales se persigue una reorganización de la estructura urbana de la ciudad, permitiendo la transformación de los espacios públicos en clave de calidad, infraestructura y servicios, el acercamiento de los servicios y la desconcentración residencial, la promoción de encuentros sociales a partir de una participación y revalorización de la vecindad y los barrios, y la mejora de la ecoeficiencia, la renovación urbana, la movilidad y el emprendimiento en la ciudad. En términos de planificación urbanística, este programa ciertamente establece una reestructuración de la trama a partir de una desconcentración residencial, de servicios y de equipamientos verdes, así como un rediseño completo de la promoción integral de La Paz previsto en el Plan general. Mientras que en este se establecía una complementariedad de los espacios urbanos y rurales, así como un diseño heterogéneo -en términos socioespaciales- de la región metropolitana, el programa de centralidades urbanas articularía un rediseño urbanizador de la ciudad, centrado en la promoción de polos de urbanidad. Además, se produce una re-territorialización de la ciudad desde arriba, desde una planificación basada en el diseño de áreas residenciales con infraestructuras propias, que no sólo entraría en conflicto con la planificación conjunta de los servicios públicos de toda la ciudad al completo, sino que abre la reflexión en torno a si esa 'recuperación de la vecindad' transmitida por el plan se restringiría a la creación de identidades de índole comunitarista reducida a dichas centralidades, o a la recuperación o reconocimiento de prácticas culturales barriales, distritales o de cualquier otra forma de prácticas de identificación socioespacial más allá de ellas. La reestructuración de La Paz a partir de estas nuevas centralidades urbanas, entonces, implicaría la proyección de un imaginario de lo urbano, vinculado a un ideal de la planificación de nuevos territorios planificados para la misma y, al mismo tiempo, a una promoción urbana en clave regional y global no a partir del conjunto de la ciudad, sino de las representaciones articuladas por estas centralidades urbanas -espacios residenciales nuevos, de calidad, con infraestructura de servicios propios, equipamientos verdes y espacios públicos nuevos y lugares de ocio renovados-.

\subsection{Imaginación cartográfica y representaciones del espacio urbano en La Paz y en Bolivia}

Una vez sintetizadas las bases del Plan Integral La Paz 2040 y un primer acercamiento a los ejes que estructuran el tipo de imaginación geográfica urbana de la Paz, este epígrafe se centrará en los elementos discursivos que construyen el "mapeado urbano" producido desde el ámbito institucional. Es decir, considerando la imagen cartográfica como "la representación cartográfica estructurada de una información espacial o seleccionada (...) que se convierte en mapa cuando se 
representa física, virtual o lingüísticamente" (Johnston et. al, 2000, p. 320), profundizaremos en algunos de los imaginarios urbanos reconocidos desde la esfera institucional a través de dicha normativa y las proyecciones realizadas desde plan acerca de representaciones cartográficas urbanas vinculadas a La Paz.

En primer lugar, encontramos que se proyecta un espacio urbano que combina la sostenibilidad y el reconocimiento de la importancia de los recursos naturales y su patrimonio hídrico, con la rehabilitación y renovación urbana, especialmente del centro de la ciudad. No se trata de una cuestión menor, pues si bien en un principio parecerían combinarse los espacios rurales y urbanos que caracterizaban al municipio, finalmente las proyecciones cartográficas pergeñadas y el diseño de las centralidades urbanas mostrarían una preeminencia de los imaginarios urbanos y urbanizantes en el conjunto de las representaciones de la ciudad.

Además, se hace un especial hincapié en el bienestar y la calidad de vida urbana, encarnada fundamentalmente por la articulación territorial en clave residencial, de trabajo y ocio, por un lado, y en la confluencia de espacios públicos ordenados -tanto en la estructuración de la trama urbana como en el diseño de la movilidad urbana, centrado en el transporte público y la peatonalidad- por otro. En este sentido, se alude tanto a la vertebración de un plan de vivienda digna -es significativo el apelativo 'de verdad' utilizado en el plan para definir los barrios-, como la previsión de servicios 'de calidad', tanto sanitarios como educativos, de ocio o de transporte, que entronca con las representaciones regionales y globales ligadas a la calidad de vida urbana. Así, se introducen algunas singularidades destacables vinculadas a La Paz dentro de ese imaginario cartográfico de calidad de vida urbana de vocación global. En primer lugar, la construcción de una 'cultura de paz' para la convivencia social pacífica y armónica paceña que se articularía no mediante el diseño de un plan de acciones punitivas como es habitual en otras normativas afectadas por el discurso del civismo urbano-, sino haciendo hincapié en la prevención y corresponsabilidad comunitaria, así como en el fomento de condiciones de diálogo y concertación (Gobierno autónomo municipal de La Paz, 2015, p. 52). Ello sería premisa para la configuración del segundo elemento distintivo de la localidad, que sería la construcción de un 'entorno feliz' a partir de la inclusión, la participación, la diversidad e interculturalidad y las condiciones equitativas de interrelación (Gobierno autónomo municipal de La Paz, 2015, p. 62). Este entorno feliz tendría su encarnación tanto en la reestructuración de las viviendas como en la construcción de las centralidades urbanas, ligando un tipo de 'entorno feliz' a los hábitos residenciales o al ocio urbano. Asimismo, y en términos de proyección de la ciudad como recurso turístico, la urbanidad se practicaría a través del imaginario de 'Ciudad maravillosa', en la que el entorno feliz y la cultura de paz se entrelazarían con la existencia de un incomparable patrimonio natural en las proximidades del municipio. En relación con todo lo anterior, emerge entonces un imaginario urbano promotor de la identidad paceña e inextricablemente unida a la misma, que combinan la diversidad y muticulturalidad contemporánea con el respeto y la revitalización del patrimonio y la memoria de las diversas expresiones culturales recogida a lo largo de la historia de la ciudad. En otras palabras: 
“[se] Promueve el respeto, valoración, protección, conservación, revitalización y restauración del patrimonio material (arquitectónico, arqueológico y natural) e inmaterial (histórico y cultural), rescata la memoria de las diversas expresiones culturales y la identidad paceña, y promueve la multiculturalidad con igualdad y diversidad de las culturas, la interculturalidad de un modo horizontal y sinérgico con diálogo, cohesión social, equidad, inclusión social y respeto" (Gobierno autónomo municipal de La Paz, 2015, p. 72).

Por último, esa relación entre la identidad paceña y los diferentes procesos territoriales a partir de imaginarios de sustentabilidad, calidad de vida, multiculturalidad seguridad y convivencia, felicidad y orden urbano particulares de La Paz se localizaría en una re-territorialización que trasciende el municipio, al impulsarse la región metropolitana de La Paz incluso más allá de sus límites locales, como hemos visto anteriormente. Esta delimitación intentaría consolidar esta región también en los entornos del Altiplano, los Valles y Amazonas, y, desde su promoción a partir de proyecciones cartográficas en clave escalar regional y global, como imaginario metropolitano de difusión y situación de la singularidad paceña de modo simultáneo a la proyección de las transformaciones llevadas a cabo en el territorio de La Paz. En ese sentido, consideramos igualmente importante acercarnos a las retóricas y valores presentes en las representaciones de lo urbano en La Paz desde su reconstrucción del sentido de lo urbano en el estado boliviano, y de lo que ello implicaría como la práctica de lo urbano de La Paz en Bolivia. Así, destacaríamos, en primer lugar, cómo las narrativas principales acerca de los usos del suelo y de la planificación territorial se vertebran en función de la capacidad productiva en el conjunto del territorio y en los usos dados a este. En segundo lugar, encontramos una continuidad narrativa en cuanto a la planificación del desarrollo integral y sustentable a largo plazo para la mejora de la calidad de vida en la ciudad y los procesos desarrollados en el Estado. Al mismo tiempo, se enfatiza la participación activa y estratégica de multitud de actores sociales entre públicos y privados -el Plan integral de La Paz 2040 contó con la participación de más de diez mil personas de la sociedad civil en su conjunto (Gobierno autónomo municipal de La Paz, 2015, p. 8)-, como elemento indisoluble de cualquier forma de planificación institucional.

En cuarto lugar, el establecimiento de imágenes objetivo del plan destaca la necesidad de las relaciones interadministrativas a distintos niveles, la importancia de la autonomía existente a nivel municipal, metropolitano y regional, o el papel de las instituciones y la participación intercultural e inclusiva como patrimonio no sólo de la ciudad, sino de Bolivia en su conjunto, reconstruyendo desde la normativa de planificación urbana la espacialidad de la práctica de horizontes valorativos asociados de La Paz hacia el resto del estado. En quinto lugar, tanto la vertebración del plan de centralidades urbanas como el desarrollo de centros de innovación y emprendimiento se legitiman en el documento en base a la situación estratégica de La Paz en el Estado boliviano, no sólo por su localización geográfica -recordemos que la expansión territorial paceña en clave de región metropolitana ya se hace atendiendo a esta variable-, sino por su centralidad política, es decir, por su importancia como motor 
económico, discursivo y político de Bolivia. De hecho, el plan, en su Introducción, sitúa dos hitos centrales que ligan la ciudad al conjunto del Estado: el primero tendría lugar en el año 2025, fecha en que se celebrará el Bicentenario de la creación de Bolivia; y el segundo se prevé para el año 2040, como preparativo para la celebración de los 500 años de la fundación de la ciudad de La Paz, que tendría lugar en el año 2048 (Gobierno autónomo municipal de La Paz, 2015, p. 8). En cuanto al primer acontecimiento, el propio plan prevé que su desarrollo se enmarque en una celebración a escala estatal, por lo cual la centralidad entre las proyecciones y representaciones sociales de la ciudad se ligará directamente a los imaginarios del bicentenario de Bolivia. Respecto al segundo, aún es pronto para aventurar las formas de promoción y proyección cartográfica urbana, pero siguiendo el vínculo existente en el plan entre la participación multicultural, la inclusión, la cultura de paz y el reconocimiento a la memoria de la diversidad cultural histórica paceña, no es descartable la recreación de una imaginación geográfica paceña que entronque no sólo los quinientos años desde su creación, sino la vertebración del Estado boliviano y diversas formas de articulación de los imaginarios de transformación y emancipación de América Latina en su conjunto.

Por último, y para completar un acercamiento a las diferentes escalas geográficas desde donde se narra la práctica de la ciudad en el Plan Integral La Paz 2040, encontramos interesante hacer alusión a lo que conceptualizaremos como una incipiente práctica de producción de globalidad (Limón López, 2018), esto es, de generación de imágenes y/o representaciones que implican la inclusión de determinados lugares en una visión mundial vinculada a los llamados procesos de globalización y que, a su vez, suponen la resignificación, contestación o negociación de tales imaginarios globales desde las localidades. En este caso, se entrelazarían la imaginación geográfica urbana paceña y la representación global de Bolivia, en una alusión explícita que abre el Plan enunciando este instrumento como herramienta para "hacer frente a los grandes cambios que están ocurriendo a nivel global con flexibilidad ante las necesidades locales emergentes en pos de la mejora de calidad de vida" (Gobierno autónomo municipal de La Paz, 2015, p. 8).

\section{Conclusiones: reconfigurando la ciudad e imaginando lo urbano}

A partir del recurso a la Geografía legal, este artículo realiza un análisis de la planificación urbana proyectada para la ciudad de La Paz (Bolivia), entre 2015 y 2040. Esta aproximación tenía como objetivo acercarnos a la imaginación geográfica contenida en el Plan La Paz 2040, de cara a contribuir a una reflexión más amplia en torno a los debates sobre la ciudad y lo urbano, y a cómo nos acercamos a estos objetos de estudio desde un estudio de caso. Igualmente, entendemos que la delimitación del objeto de análisis, -esto es, un plan integral que rige la ciudad de La 
Paz durante 25 años analizado desde el aislamiento del contexto de su aplicación- se beneficiaría de un diálogo con otros análisis más completos sobre el municipio en particular y sobre la cuestión urbana en Bolivia, en general ${ }^{4}$. Sin embargo, lo planteamos como una posibilidad de acercamiento al caso que pueda entenderse como generador de hipótesis de cara a una mayor profundización y ampliación de esta investigación, centrada en la práctica de la ciudad en el municipio de La Paz a través de la imaginación geográfica urbana que encontramos en su planificación urbanística.

Desde esa perspectiva, hemos revisado los patrones de ocupación del territorio presentes en la normativa, que es objeto de una regulación exhaustiva, normativa y que establece una nueva división territorial en el municipio. Pese a la identificación como rural de un elevado porcentaje del territorio municipal y de un uso agrícola de la mitad de su suelo, el plan propone una redistritación de la ciudad en torno a un programa para establecer 19 centralidades urbanas jerárquicas, proyectadas como polos de desarrollo urbano. Esta proyección, entonces, desde la potencialidad urbanizadora de las centralidades, delimita las formas socioespaciales adscritas a la práctica de nueva delimitación del territorio municipal a partir de su relación con estas nuevas entidades, concebidas como productoras de urbanidad desde las que aproximarse también al entorno rural (hay centralidades urbanas y rurales, y el plan remite a ambas). Estas centralidades serían proyectadas también como nodos de interacción social, donde determinadas prácticas socioculturales son definidas y visibilizadas como parte de ese tipo ideal de ciudad. En una formulación que recordaría a la teoría de los lugares centrales propuesta por Christaller en 1933, el plan reconfiguraría la ciudad y sus límites territoriales desde una visión policéntrica, elemento común en la práctica urbanística de otras ciudades de la región y en las investigaciones sobre cuestiones urbanas en América Latina.

Por otra parte, nos hemos acercado a los imaginarios locales urbanos proyectados en el plan, y que abarcarían desde una cultura de paz hasta la multiculturalidad y diversidad, y que sostendrían una representación de La Paz a partir de las formas de expresión de estos valores como parte de la urbanidad paceña. De hecho, como vimos en la sección 3.2, el documento define ciertas prácticas desarrolladas en torno a la construcción de la planificación urbana del municipio como valores distintivos y diferentes de La Paz, estableciendo los límites de una práctica de ciudad y la naturaleza de las actividades propias del espacio público urbano. Esas expresiones de lo urbano (participación intercultural, por ejemplo) son marcadas como referentes de demarcación del municipio y, al tiempo, con vocación de trascender la ciudad, para vertebrar un espacio definido por prácticas específicamente asociadas a La Paz. En ese sentido, el Plan 2040 establece la ciudad añorada, La Paz que queremos, articulada desde una representación específica de lo urbano en Bolivia. Finalmente, esa ciudad se significa en todas las escalas geográficas en su normativa, desde lo local

\footnotetext{
${ }^{4}$ Para una revisión completa y actualizada de las investigaciones sobre cuestiones urbanas en Bolivia ver, por ejemplo, Urquieta, 2016.
} 
a lo regional-metropolitano, como hemos visto, y también respecto al Estado boliviano y a una imaginación de los procesos de globalización que hace necesaria su reestructuración, elemento común en los instrumentos de planificación urbanística de los últimos años. En otras palabras, el plan integral demarca la práctica de una concepción de lo urbano no sólo en el territorio municipal sino que localiza en La Paz como una ciudad ligada a valores específicos presentes en diferentes escalas desde los que comprender la planificación urbanística.

En definitiva, entendemos que este análisis normativo obliga a una cierta reflexión sobre las formas en las que investigamos lo urbano y la ciudad; por una parte, un acercamiento a la práctica de esas ciudades nos permite un primer acercamiento a las formas socioespaciales heterogéneas (o definidas como rurales y urbanas) que nos encontramos en el entorno de la ciudad, y, sobre todo, a las maneras de regularlas en términos de ocupación del territorio, que tienden a continuar esa delimitación que responde a un tipo ideal entre ambas formas sin abrir camino a diferentes maneras de conceptualizar continuidades espaciales de formas heterogéneas. Por otra parte, esta lectura de la normativa apunta a que la heterogeneidad de prácticas que ocurren en el espacio de la ciudad tienden a ser reguladas específicamente como formas de expresión propias de lo urbano, que se define como un sistema propio, a reordenar en base a su propia autoreferencialidad. Sería interesante, por ejemplo, profundizar sobre qué prácticas previas son promovidas e identificadas como parte de la interacción social ligada a una nueva distritación urbana, por su importancia como elemento de reflexión sobre las representaciones de lo urbano en Bolivia. Al mismo tiempo, las narrativas establecidas en el plan respecto a la centralidad del municipio en el Estado boliviano y a su proyección en diferentes escalas geográficas sugerirían una formulación de la ciudad como nodo de valores políticos distintivos ligados a la práctica de lo urbano; en definitiva, como una representación ideológica de los procesos de urbanización. Todas estas ideas iniciales abren un espacio para generar hipótesis preliminares que, aunque exceden el objetivo de este trabajo iniciático, forman ya parte de un universo de investigación en torno a la práctica de la ciudad y al objeto de estudio de las investigaciones sobre cuestiones urbanas.

\section{Referencias bibliográficas}

Allen, J. y Massey, D. (eds.) (1995): Geographical Worlds. New York, Oxford University Press.

Banakar, R. (2010). Studying the rights discourse: a tentative socio-legal framework. En B. Lemann (ed.) The Nordic Sociology of Law, ( $1^{\mathrm{a}}$ edición) DJOF, Copenhague.

Banakar, R. (2011). The sociology of Law: from industrialisation to Globalisation. Working Paper, 11-03, University of Westminster, pp. 1-32.

Baptista, I. (2013). Practices of exception in urban governance: reconfiguring power inside the State. Urban Studies, 50 (1), 39-54.

Barkan, J. (2011). Law and the geographic analysis of economic globalization. Progress in Human Geography, 35 (5), 589-607. 
Blomley, N. (2003a). Law, property and the spaces of violence. Annals of the Association of American Geographers, 93 (1), 121-141.

Blomley, N (2003b): Unsettling the city: urban land and the politics of property ( $1^{\mathrm{a}} \mathrm{ed}$.). New York, Routledge.

Blomley, N (2004). Un-real estate: proprietary space and public gardening. Antipode, 36 (4), 614-641.

Blomley, N (2008). Making space for law. En K. Cox, M. Low \& J. Robinson. The Sage Handbook of political geography (pp. 155-168). London, Sage

Blomley, N (2010). The right to pass freely: circulation, begging and the bounded itself. Social and Legal Studies, 19 (3), 331-350.

Bennett, L. y Layard, A. (2015). Legal Geography: becoming spatial detectives. Geography Compass, 9 (7), 406-422.

Braverman, I., Blomley, N.delaney, D. y Kedar, A. (2013). The expanding spaces of law: a timely Legal Geography. Buffalo Legal Studies Research Paper Series, 2013-032, 1-29.

Burawoy, M. (1991).Ethnography unbound. Power and resistance in the modern metropolis. Berkeley-Oxford: University of California Press.

Chouliaraki, L. y Fairclough, N. (1999): Discourse in Late Modernity: Rethinking critical discourse analysis. Edinburgh, Edinburgh University Press.

Cox, K.; Low, M. y Robinson, J. (2008): The Sage Handbook of political geography. London, Sage.

Dangschat, J. S. (2009). Space matters. Marginalization and its places. International Journal of Urban and Regional Research, 33 (3), 835-840.

Delaney, D. (2015a), Legal Geography I: constitutivities, complexities and contingencies. Progress in Human Geography, 39 (1), 96-102.

Delaney, D. (2015b). Legal Geography II: discerning injustice. Progress in Human Geography, DOI: $10.1177 / 0309132515571725,1-8$.

Denzin, N. y Lincoln, Y. (2005). The Sage Handbook of qualitative research. LondonThousand Oaks-New Delhi, Sage.

Forest, B. (2004). The legal (de) construction of geography: race and political community in Supreme Court redistricting decisions. Social and Cultural Geography, 5 (1), 55-73.

Gregory, D.; Johnston, R.; Pratt, G.; Watts, M. J. y Whatmore, S. (eds.) (2009). Diccionary of Human Geography ,5th Edition. Malden:Blackwell.

Gobierno Autónomo Municipal de La Paz (2015) Plan Integral La Paz 2040. Plan de desarrollo municipal. Plan Municipal de Ordenamiento territorial, La Paz: SPC.

Herbert, S. (2010). Contemporary geographies of exclusion III: to assist or punish? Progress in Human Geography, 35 (2), 256-263.

Instituto Nacional de Estadística (2018), recuperado de www.ine.gob.bo (14 de febrero de 2019)

Johnston, R. J.; Gregory, D. y Smith, D. (2000). Diccionario Akal de Geografía Humana, (1 ${ }^{\mathrm{a}}$ ed.) Madrid:Akal

Ley Marco de Autonomías y Descentralización (Ley número 031) “Andrés Ibáñez”, Gaceta Oficial del Estado Plurinacional de Bolivia, La Paz, 19 de julio de 2010 
Ley Municipal Autonómica Número 068 del Gobierno autónomo municipal de La Paz, donde se aprueba la formulación del Plan de Desarrollo Municipal Integral 'Plan La Paz 2040', Gaceta Oficial del Estado Plurinacional de Bolivia, La Paz, 4 de abril de 2014.

Limón López, P. (2018). Gobernanza metropolitana y producción de globalidad en Madrid y Barcelona. Gapp. Nueva Época, 20, 65-81.

Lippens, R. (ed.) (2004). Imaginary boundaries of justice: social justice across disciplines. Oxford:Hart Publishing.

Martin, D.; Scherr, A. W. y Christopher C. (2010). Making law, making place: lawyers and the production of space. Progress in Human Geography, 34 (2), 175-192.

Urquieta, P. (2016). Avances y desafíos de la investigación y la formación en temas urbanos en Bolivia. En P. Metzger, J. Rebotier, J. Robert, P. Urquieta y P. Vega Centeno (eds) (2016) La cuestión urbana en la región andina (pp.21-75) Quito: Pontificia Universidad Católica del Ecuador.

Mitchell, D. (1997). The annihilation of the space by law: the roots and implications of antihomeless laws in United States. Anthipode, 29 (3), 303-335.

Mitchell, D. (2003). The liberalization of free speech: or, how protest in public space is silenced, Stanford Agora, September 2003. Disponible en http://agorastandford.edu/agora/volume4/articles/mitchell/mitchell.pdf

Mitchell, D. y Staeheli, L. (2005). Permitting protest: parsing the fine geography of dissident in America. International Journal of Urban and Regional Research, 29 (4), 796-813.

Mitchell, D. y Heynen, N. (2009). The geography of survival and the right to the city: speculations on surveillance, legal innovation and the criminalization of intervention. Urban Geography, 30(6), 611-632.

Scott, A.J. y Storper, M. (2014). The nature of cities: The Scope and Limits of Urban Theory. International Journal of Urban and Regional Research, 39(1), 1-15.

Staeheli, L. A. (2010). Political geography: democracy and the disorderly public. Progress in Human Geography, 34 (1), 67-78.

Young, D. y Keil, R. (2010). Reconnecting the disconnected: the politics of infrastructure in the in-between city. Cities, 27, 87-95.

Wachsmuth, D. (2014). City as ideology: reconciling explosion of the city form with the tenacity of the city concept. Environment and Planning D: Society and Space, 32 (1), 7590 . 